Revue européenne des sciences sociales

European Journal of Social Sciences

XLIV-134 | 2006

Quel(s) défi(s) pour les sciences sociales à l'heure de la mondialisation?

\title{
La raison distribuée. Genèse des concepts collectifs dans la modélisation mathématique en sciences sociales
}

Henri Volken

\section{(2) OpenEdition}

\section{Journals}

Édition électronique

URL : http://journals.openedition.org/ress/318

DOI : $10.4000 /$ ress.318

ISSN : $1663-4446$

\section{Éditeur}

Librairie Droz

\section{Édition imprimée}

Date de publication : 1 juin 2006

Pagination : 301-315

ISBN : 9-782-600-01095-5

ISSN : 0048-8046

\section{Référence électronique}

Henri Volken, «La raison distribuée. Genèse des concepts collectifs dans la modélisation

mathématique en sciences sociales », Revue européenne des sciences sociales [En ligne],

XLIV-134 | 2006, mis en ligne le 15 octobre 2009, consulté le 04 mai 2019. URL : http:// journals.openedition.org/ress/318; DOI : 10.4000/ress.318 


\section{Henri VOLKEN}

\section{LA RAISON DISTRIBUÉE. GENÈSE DES CONCEPTS COLLECTIFS DANS LA MODÉLISATION MATHÉMATIQUE EN SCIENCES SOCIALES}

Sciences sociales et mathématiques n'ont pas toujours fait bon ménage. Et pourtant elles peuvent s'influencer mutuellement de manière intéressante. Nous allons essayer de retracer quelques étapes de leur histoire commune et en particulier montrer comment le passage de concepts individuels à des notions plus «sociales» a permis la construction de modèles de certains comportements collectifs qui ont une pertinence aussi bien dans les sciences sociales que dans le domaine des mathématiques et de la physique.

\section{APRÈS GALILÉE}

Tout commence avec Galilée. Du moins en ce qui concerne la physique et son prodigieux envol depuis le dix-septième siècle. Un envol tel, qu'au vingtième siècle, elle a été perçue sans conteste comme la science dominante. Cela aussi bien pour les résultats décisifs et spectaculaires qu'elle a obtenus que pour sa méthode rigoureuse, constituée d'un mélange subtil d'approche expérimentale faite de technicité raffinée et de formalisation hautement abstraite. C'est la physique qui a apporté la plus forte contribution à la compréhension du monde, aussi bien dans la cosmologie que dans le domaine de l'infiniment petit, où une forme inquiétante d'incertitude semble prévaloir. La théorie du Big Bang, les développements récents de l'astrophysique et la physique quantique constituent des théories redoutablement précises et fécondes. Elles forment aussi le cadre d'une interrogation épistémologique et philosophique ouverte dont l'intérêt dépasse de loin la communauté des physiciens et touche tous les domaines du savoir. La physique a fait rêver et réfléchir durant la plus grande partie du siècle passé.

Mais quel est exactement le mérite de Galilée dans ce développement? C'est probablement avec Galilée que les mathématiques ont commencé à jouer un rôle capital dans l'organisation théorique d'une science. Tout d'abord, elles fournissent un langage formalisé, univoque et opérationnel qui oblige les scientifiques à préciser leurs idées, parfois même dans une douloureuse exigence de clarté formelle. Mais cela a l'avantage de permettre l'échange des idées, la confrontation des arguments et l'identification rapide des points obscurs ou litigieux. Mais surtout - et c'est le second apport essentiel - les mathématiques fournissent des méthodes efficaces, sans cesse renouvelées, pour résoudre des problèmes transcrits dans leur formalisme. Pour cela elles bénéficient d'une histoire très ancienne et d'un corpus largement cumulatif. Ainsi le théorème de Pythagore est utilisé dans tous les calculs qui permettent la localisation spatiale. Il n'y a donc pas de vol 
d'avion, pas de mission spatiale, mais pas non plus d'architecture sans lui. Et pourtant ce résultat a été obtenu et inscrit dans la mémoire mathématique il y a plus de deux mille ans. Il figure essentiellement sous la même forme dans nos manuels actuels.

A partir du moment où les mathématiques sont entrées dans la pratique de la physique, celle-ci s'est développée de manière beaucoup plus systématique et organisée. Son domaine s'est à la fois simplifié et précisé. Mais surtout, cela a engendré d'innombrables questions nouvelles et permis d'échafauder des théories et les moyens de les vérifier. Le fait de ne pas dépendre exclusivement de l'expérimentation pour accroître le savoir scientifique, mais de pouvoir dériver rigoureusement des résultats à partir d'autres résultats en se basant sur les propriétés des objets mathématiques utilisés pour représenter des phénomènes réels, est à l'origine d'une extension immense et cohérente du savoir physique. Parfois ces objets formels ont dû être inventés pour résoudre des problèmes spécifiques. C'est le cas des infiniment petits, introduits indépendamment par Leibniz et Newton. C'est le début de l'analyse mathématique et l'introduction des notions de dérivée et d'équation différentielle, outils essentiels pour l'étude de la dynamique des systèmes.

Avec Newton, la physique a atteint l'un de ses sommets. Avec un minimum de concepts - qui doivent beaucoup à Galilée - avec quelques rares principes d'une grande élégance et simplicité, Newton a pu poser les bases de la mécanique. C'est la mécanique d'aujourd'hui, même si elle a été généralisée depuis par la théorie de la relativité d'Einstein. Dans son système, il devient possible de décrire avec précision la trajectoire d'un objet dans l'espace. Par conséquent de prédire le futur de son évolution. Cela a eu des conséquences majeures sur la compréhension de notre système planétaire, mais aussi sur le développement de la technologie et, plus tard, sur l'essor de l'industrie. Mais à côté de cela, le système de la mécanique de Newton est à l'origine d'un optimisme grandissant sur les possibilités d'appliquer cette méthodologie - considérée comme le prototype de la pensée scientifique - à d'autres domaines du savoir. Notamment aux domaines qui correspondent aujourd'hui aux sciences sociales et aux sciences politiques.

\section{LÉVIATHAN}

C'est à la suite des découvertes de Galilée sur les équations du mouvement que Hobbes propose dans son œuvre majeure, Léviathan, une vision mécaniste de la manière de gouverner. Pour proposer une théorie politique extraordinairement ambitieuse et purement rationnelle, Hobbes espère utiliser la même rigueur logique et la même économie dans l'usage des concepts que Galilée dans sa description des lois du mouvement. Il adopte pour cela une attitude proche de la démarche axiomatique des mathématiciens et tente de déduire logiquement sa théorie à partir de quelques rares principes qui lui paraissent indiscutables. Le principe premier et fondamental introduit par Hobbes est la volonté de pouvoir, principe atténué dans sa portée par des principes secondaires introduisant la notion 
de coopération par exemple. A partir de ces quelques principes, la théorie se construit déductivement, à la manière de la géométrie des mathématiciens grecs. Hobbes était arrivé tardivement à s'intéresser aux mathématiques, et d'après certaines sources, c'est en lisant les éléments d'Euclide qu'il s'était passionné pour cette démarche scientifique particulière et pour la rigueur de la pensée déductive en général. Aujourd'hui, nous nous étonnons de cette entreprise et de la tentative de réduire l'homme à un automate mécaniste et la pensée à des algorithmes. Mais, au cours du dix-septième siècle, on y voyait un espoir évident de progrès, ce que montre clairement l'utilisation de la métaphore de l'horloge, qui se retrouve de manière itérée chez de nombreux philosophes. La science semblait une possibilité, la seule peut-être, de sortir d'un certain obscurantisme et d'atteindre les lumières.

La tentative de Hobbes, aussi peu souhaitables que nous paraissent aujourd'hui ses implications, est un exemple important d'une approche scientifique des sciences humaines dans laquelle le but est de décrire - à partir de principes généralement admis - le monde social tel qu'il est, et non pas tel qu'il devrait être. Bien sûr, on peut avoir des avis divers sur la réussite de l'opération, mais l'approche de Hobbes reste remarquable par sa précision logique et son éloignement de toute position normative. Mais la réaction qu'elle a suscitée représente aussi l'image typique de la peur que peut inspirer une approche scientifique des sciences sociales et des sciences humaines en général par des moyens formels.

Qu'en est-il aujourd'hui? Les sciences formelles et les sciences physiques ont-elles quelque chose à dire dans ces domaines? Nous allons tenter de montrer que les mathématiques et la physique sont elles-mêmes en partie des sciences «sociales», dans un sens très modeste, et peuvent par conséquent apporter un point de vue constructif à la discussion des phénomènes humains et sociaux.

\section{THERMODYNAMIQUE ET COLLECTIVITÉS}

Le dix-septième siècle perçoit les progrès de la physique notamment à travers les réussites de Galilée et Newton, avec leur argument décisif: la prédiction, rendue possible grâce à la simplicité et l'élégance de l'appareil conceptuel et à l'efficacité du langage mathématique utilisé. On peut enfin expliquer la chute des corps sans les complications souvent obscures et les incohérences de la physique d'Aristote. On comprend mieux le mouvement des corps célestes, on prévoit avec facilité leurs positions le long des orbites. La science a gagné un prestige et un pouvoir immenses. Il n'est pas étonnant que l'idée d'appliquer de si performants outils à leur propre science ait effleuré de nombreux scientifiques des domaines «humains » au cours des dix-septième, dix-huitième et dix-neuvième siècles. On assiste à la naissance de la statistique (mot construit au dix-huitième siècle à partir de «Staat», allusion à la description ou étude quantitative des états), à la mathématique sociale du marquis de Condorcet (1922) et à de nombreuses applications plus ou moins convaincantes des méthodes «dures» dans des sciences qui le sont un peu moins. L'influence de la démarche de Newton se retrouve aussi 
bien chez Locke, Hume ${ }^{1}$ et Smith que chez Desaguliers ${ }^{2}$ et Quesnay, qui est à l'origine du mouvement physiocrate, et de nombreux autres auteurs.

Or l'enthousiasme pour les méthodes de la mécanique de Newton s'est heurté, dans le domaine même de la physique, à une situation inattendue. Sous l'impulsion des chimistes, dont les méthodes devenaient de plus en plus scientifiques, les physiciens se sont penchés sur le problème d'intégrer les gaz dans leurs théories. Il était admis, depuis le milieu du dix-huitième siècle, que les gaz étaient constitués de petites particules en mouvement. Les anciennes théories atomistes, remontant à Leucippe et Démocrite, étaient à nouveau admises, et aussi bien Galilée que Newton croyaient à la réalité des atomes comme constituants de la matière. Rien ne s'opposait en principe à traiter les atomes individuels comme particules en mouvement et donc de leur appliquer les équations de Newton pour décrire leurs trajectoires. Or si théoriquement cela semblait possible, les milliards d'atomes que l'on supposait constituer la moindre quantité de gaz rendaient impossible tout calcul de ce genre. L'état gazeux et sa dynamique échappaient apparemment à l'emprise des équations. Ce problème a été indirectement à l'origine d'un débat épistémologique capital, celui du déterminisme. Comment interpréter notre incapacité de calculer le mouvement des atomes dans un gaz? Est-ce une simple limitation due à la quantité de calculs et de mesures à effectuer, ou est-ce une réelle barrière fondamentale impossible à franchir?

Les nouveaux développements de la chimie ont montré comment cette question pouvait en fait être contournée par une approche toute différente. De nouveaux concepts ont été introduits, comme la pression et la température, dont le lien avec le mouvement des atomes n'était plus très clair. En fait il s'agissait de phénomènes plus globaux, plus collectifs, mais observables et mesurables au niveau macroscopique. Puisque la nature de ces phénomènes ne pouvait pas s'expliquer par des propriétés individuelles des atomes, il fallait trouver d'autres méthodes, d'autres structures mathématiques pour les décrire. L'étude des propriétés de la chaleur et le développement d'un arsenal conceptuel radicalement nouveau devenaient primordiaux. La palette de ces concepts incluait des notions statistiques et probabilistes. La thermodynamique était née.

De manière parallèle, les tentatives d'application de méthodes mathématiques pour étudier les sociétés humaines rencontraient le même problème. On pouvait mesurer des caractéristiques prélevées sur des individus, les comparer, les intégrer dans des calculs, sans pour autant être capable de montrer le lien de ces traces numériques avec des phénomènes collectifs, économiques ou sociaux, que l'on observait à une autre échelle. Cette difficulté était de divers ordres. Très souvent, le nombre considérable d'individus impliqués dans des interactions sociales rendait les mesures et les calculs impraticables. Mais de manière plus fondamentale, aucune théorie causale ne parvenait à montrer la dépendance des

«... that politics may be reduced to a science», in S. Copley \& A. Edgar (eds), Selected Essays, Oxford, Oxford University Press, 1993.

2 Comme en témoigne le titre anglais de son livre: The Newtonian System of the World; The Best Model of Government, an Allegorical Poem (1728); trad. française et commentaires: Jean-Théophile Desaguliers, un Huguenot, philosophe et juriste, en politique, Paris, Honoré Champion, 1999. 
phénomènes émergents avec les propriétés individuelles. Curieusement, au lieu d'imiter le chemin emprunté par les chimistes et les physiciens dans l'étude des gaz, la faute a été attribuée, dans le cas de la méthodologie des sciences sociales, au domaine lui-même, qui ne se «prêtait» pas aux applications des mathématiques. Ce qui pouvait être une forme de résistance comparable à celle qui invoquait le vitalisme dans le domaine de la biologie.

Or c'est précisément dans la préoccupation des scientifiques pour trouver une théorie satisfaisante pour l'étude des gaz et de la chaleur que se profile une nouvelle manière de penser. C'est à Sadi Carnot, dont la courte carrière s'est déroulée dans la première moitié du dix-neuvième siècle, que l'on doit la première théorie physique «collective», la thermodynamique. Cette théorie abandonnait l'idée de traiter individuellement les atomes à l'aide des équations de Newton, bien que la chaleur fût conçue, dès la fin du dix-huitième siècle, comme un mouvement de particules. Car ce mouvement est un mouvement d'ensemble, un mouvement collectif qui nécessite un traitement différent, ainsi que des objets mathématiques et des concepts nouveaux pour le décrire. La thermodynamique s'efforce de décrire des phénomènes émergents, qui, bien que provoqués par des mouvements microscopiques, individuels, ne s'expliquent pas par ceux-ci. Comment ne pas penser ici à certaines manifestations sociales comme l'apparition de panique dans une foule, qui ne s'explique pas non plus par la somme des comportements individuels? La thermodynamique a trouvé immédiatement des applications dans les nouvelles technologies comme les machines à vapeur. Mais son importance est peut-être surtout théorique. Ses trois principes fondamentaux ont eu, et ont encore, une grande importance dans les discussions épistémologiques et philosophiques autour des notions de déterminisme, de la définition de la vie et de l'avenir de l'univers. En particulier, le deuxième principe dans lequel apparaît la notion d'entropie est ubiquiste dans les débats scientifiques.

La portée de ce deuxième principe de la thermodynamique est considérable. Il implique en particulier que le flux de la chaleur est irréversible, donc que le temps possède une direction privilégiée, en accord avec notre perception, alors que les équations de Newton sont parfaitement réversibles. Mais surtout, la notion d'entropie, introduite par Clausius, constitue une étape importante vers une vision plus globale des phénomènes physiques. L'entropie est une notion abstraite, mais parfaitement définie et mesurable, qui peut être interprétée pour nos besoins et de manière simplifiée comme une mesure du désordre d'un système. Le deuxième principe de la thermodynamique peut se résumer par l'expression: dans un système fermé (c'est-à-dire sans influence extérieure), l'entropie augmente. Cette augmentation est la conséquence de l'irréversibilité du flux de chaleur. Ce qui signifie que l'univers tend vers un état d'entropie maximale, une sorte de «mort par la chaleur», ou «Hitzetod» dans la terminologie de Helmholtz.

Par analogie, ce deuxième principe de la thermodynamique, et particulièrement le concept d'entropie, a acquis une grande importance dans les applications des mathématiques et de la physique dans les sciences humaines et sociales. En particulier c'est le point de vue «thermodynamique» qui a orienté la recherche vers les phénomènes émergents et, plus tard, vers l'auto-organisation des systèmes collectifs, ce qui a profondément influencé la manière dont on perçoit actuellement l'apport des mathématiques dans ces domaines. De l'élégante 
simplicité de la mécanique de Newton, on a passé à une approche plus structurelle et moins linéairement causale, plus proche des mécanismes observés en sciences humaines.

En ce qui concerne la thermodynamique, la question restait ouverte de savoir comment déduire l'essentiel des caractéristiques du mouvement collectif à partir du mouvement des atomes dont clairement ce mouvement d'ensemble dépendait. C'est l'Ecossais Maxwell qui le premier a proposé une solution étonnante: il n'est pas nécessaire de connaître tous les détails des mouvements des atomes individuels, mais uniquement les caractéristiques d'un mouvement moyen ${ }^{3}$. Ce point de vue original introduit la théorie de la probabilité dans l'arsenal mathématique utilisé pour décrire des phénomènes physiques. Du même coup, la statistique se transforme. A son rôle de résumer de grandes quantités de données et de les rendre « lisibles», elle ajoute celui qui consiste à étendre les notions individuelles à des collectivités grâce à des concepts nouveaux comme les mesures de tendance centrale et surtout la distribution normale qui joue un rôle clé pour les applications dans les sciences sociales. La théorie cinétique des gaz et la mécanique statistique sont nées de ce point de vue élargi et marquent l'avènement des concepts collectifs en physique. Cela a une grande importance pour le développement de la méthodologie formelle dans les sciences humaines telle qu'elle est proposée aujourd'hui.

Il est toujours dangereux de faire des parallèles entre le monde des objets physiques et les sociétés humaines, les objections sont immédiates et nombreuses. Néanmoins, l'avènement de la thermodynamique marque un tournant dans le choix conceptuel et introduit des notions collectives qui semblent indiquer une parenté avec certains aspects de l'étude de phénomènes sociaux. L'un des points de rencontre est sans doute la notion de transition de phase. Comment l'eau passet-elle de l'état liquide à l'état gazeux de vapeur d'eau? Dans les deux états, les particules sont identiques et en mouvement. Mais dans le cas de la vapeur d'eau, le mouvement moyen est plus rapide, et la place occupée par chaque molécule est beaucoup plus grande. Ce mouvement moyen est mesuré par la température. En chauffant l'eau, la vitesse moyenne augmente de manière continue et l'état reste liquide. Mais pour une certaine température, l'état de la matière est de nature différente, il est gazeux. Ce changement se fait très brutalement. Cela évoque le phénomène de l'apparition de la panique dans une foule que nous avons brièvement évoquée, où l'agitation joue le rôle de la température, du moins de manière métaphorique. Mais rien ne permet de prédire, à partir des comportements individuels, le moment précis de l'explosion de la panique, bien que celle-ci dépende de manière complexe des comportements des individus. Ce phénomène de changement qualitatif brusque est fréquent dans le domaine des sciences sociales. Mais tout changement brusque n'est pas une transition de phase. Ce qui est caractéristique de ce phénomène, c'est qu'il a lieu globalement et presque instantanément,

Il faut noter à ce propos que, sur ce point, ce sont probablement les sciences sociales qui ont influencé la physique, puisque l'usage de la statistique était l'apanage des scientifiques qui étudiaient les phénomènes de société: ceux qu'on nommerait aujourd'hui démographes, politologues ou sociologues. A l'origine, la statistique était une science sociale. 
provoqué par l'interaction des individus qui composent le système, sans explication causale directe reliant ces derniers au phénomène émergent. Les méthodes développées à partir des tentatives d'explication du processus de transition de phase par la thermodynamique pourront être appliquées à des problèmes sociaux qui ont les mêmes caractéristiques générales: grand nombre d'individus et apparition de structures globales soudaines.

C'est donc l'étude des gaz et les diverses tentatives des scientifiques d'intégrer ces résultats dans le cadre explicatif de la théorie physique qui a permis de développer des notions plus « collectives ». Ces notions rapprochent les domaines apparemment si éloignés que sont les sciences naturelles et les sciences sociales. Dans ce contexte, il faut parler du concept important d'équilibre. Dans un premier temps, la thermodynamique s'est centrée sur l'étude des systèmes en équilibre thermique. Le principe de Gibbs stipule que, dans un état d'équilibre, le système s'organise pour minimiser son énergie. L'état d'équilibre est caractérisé par le fait que l'entropie ne s'accroît pas. Mais cela ne permet pas de comprendre la nature des changements à l'intérieur d'un système fermé, et surtout ne permet pas de décrire correctement les états d'un système hors équilibre. Dans la nature, de nombreuses structures correspondent cependant à des états qui ont une certaine stabilité ${ }^{4}$ sans être des états d'équilibre. C'est le cas dans la plupart des systèmes biologiques et très certainement dans tous les systèmes économiques et sociaux d'une certaine ampleur. Quel est le devenir d'un système politique nouvellement adopté? Quelles vont être les conséquences de mesures économiques? En général, comment un système hors équilibre choisit-il l'un des états stables dans lequel il va se placer provisoirement? Il n'existe pas de critère indiscutable pour définir l'attrait de ces états et par conséquent la question n'a pas de réponse immédiate.

L'étude des systèmes hors équilibre a été favorisée par le paradoxe de l'apparente incompatibilité entre thermodynamique et biologie. Comment expliquer le phénomène de la vie en présence du deuxième principe qui affirme que l'entropie d'un système ne peut que croître, c'est-à-dire que le désordre ne peut qu'augmenter? Dans tous les processus qui touchent à la vie ou aux activités humaines, s'observe une apparition, ou souvent le maintien, de l'ordre sur une durée non négligeable. C'est finalement la notion de système dissipatif, rendue populaire par Prigogine, mais basée sur des idées et des modèles mathématiques qui remontent à Poincaré, qui a permis une généralisation suffisante de la thermodynamique pour rendre compte des phénomènes biologiques, économiques et sociaux. Les systèmes dissipatifs échangent de l'énergie avec leur entourage, et permettent d'expliquer, malgré l'accroissement global de l'entropie, l'apparition de l'ordre et l'existence de structures stables. Le livre de D'Arcy Thompson (1994) donne un grand nombre d'exemples de genèse de ces formes dans la nature, qui résultent d'un état stable loin de l'équilibre.

Les premières illustrations simples de ce qu'on appelle actuellement des systèmes dissipatifs ont été découvertes par le physicien allemand Quincke mais

Steady states dans la terminologie anglaise. 
redécouvertes et décrites quelques dizaines d'années plus tard par Bénard au début du vingtième siècle. Lorsque l'on chauffe de l'eau dans un récipient sous forme de parallélépipède, on constate tout d'abord une montée désordonnée de molécules agitées par l'apport de chaleur, qui dissipent l'énergie vers le haut, puis redescendent. Mais progressivement le mouvement, jusque-là anarchique, s'organise, les molécules semblent coordonner leur mouvement et il s'installe une structure stable sous forme de rouleaux réguliers. Les molécules «coopèrent». Le système s'est auto-organisé hors de l'équilibre, dans un processus qui possède une persistance qui le rend facilement observable. C'est le cas aussi lorsqu'une foule suffisamment dense se déplace dans les deux sens sur un trottoir. Au début du processus - supposons que l'on ouvre le passage à la foule - règne une grande confusion, et de nombreuses collisions ont lieu, puis progressivement s'installe un ordre presque régulier: chaque moitié du trottoir est dédiée à l'un des sens de déplacement. Cette structure apparaît sans qu'elle soit imposée et sans concertation. Elle peut se présenter sous deux formes symétriques sans que l'on puisse prévoir laquelle de ces deux solutions s'imposera. Il s'agit d'un cas simple de point de crise, où le système «doit prendre une décision». Ce point de crise est appelé par les scientifiques un point de bifurcation. C'est un phénomène typique des systèmes hors équilibre. C'est donc un concept important pour les applications des nouvelles méthodes formelles dans les sciences humaines.

\section{VERS DES CONCEPTS SOCIAUX}

Mais comment adapter ces idées, qui proviennent des sciences naturelles et qui s'appliquent en premier lieu aux objets simples, inanimés, pour décrire, expliquer et comprendre raisonnablement des phénomènes mettant en scène des personnes douées d'intention, de volonté, de discernement et de libre-arbitre? Avant de répondre à cette question, nous allons nous intéresser à des formes de vie primitives, mais chez lesquelles on trouve une composante sociale que nous pouvons interpréter à l'aide des concepts introduits jusqu'ici. Il s'agit d'un unicellulaire microscopique vivant dans la vase, appelé Dictyostelium discoideum. Dans les périodes fastes, lorsque la nourriture est abondante et que les conditions thermiques et hydriques sont satisfaisantes, chacun de ces unicellulaires vit de son côté sa propre vie individuelle. Leur dispersion spatiale est grande, uniforme et régulière. Mais lorsque les conditions de survie deviennent plus difficiles, ils adoptent soudain et spontanément un comportement social tout différent. Ils se rapprochent, s'agglutinent par dizaines ou centaines de milliers pour former ce qui peut paraître un nouvel organisme. Ils passent par une transition de phase qui les fait passer d'une collection d'individus à une collectivité organique. Cette nouvelle structure est obtenue par auto-organisation du système, initiée localement par des signaux chimiques, mais sans qu'il y ait une forme de contrainte centralisée. Ce nouvel organisme se comporte alors comme un individu cohérent à une échelle supérieure, se déplace, prend parfois racine et change éventuellement de forme. Mais, fait plus surprenant, les unicellulaires qui composent ce nouvel organisme se comportent comme des cellules d'un organisme et se spécialisent. Certaines de ces cellules se regroupent et se transforment en spores, c'est-à-dire des cellules au métabolisme presque nul, capables de survivre à des 
conditions très hostiles et d'assurer ainsi la survie de l'espèce jusqu'à ce que les circonstances extérieures redeviennent plus favorables.

Ce dernier exemple montre l'émergence d'un organisme macroscopique, avec une spécialisation de ses constituants à partir d'une population uniforme d'unicellulaires microscopiques. Ce phénomène social se réalise sans intervention centralisée, uniquement par des ajustements locaux, de nature très primitifs, entre les individus. Ces ajustements, provoqués par des substances chimiques, constituent la seule forme de communication. C'est un exemple de structure sociale émergente, laquelle ne peut pas s'expliquer uniquement par la connaissance des comportements individuels, mais qui dépend de manière complexe des interactions entre individus ainsi que des interactions entre individus et environnement.

Le même phénomène se retrouve chez des animaux plus évolués, les poissons et les oiseaux par exemple. Il n'est pas rare de voir des petits poissons se regrouper en un banc qui suggère l'apparence d'un poisson beaucoup plus grand et semble se mouvoir comme tel. Or aucun des petits poissons n'a de vue d'ensemble, ni ne connaît les règles de cette étrange chorégraphie qui peut probablement éloigner ou décourager un certain nombre de prédateurs. La structure macroscopique et la cohérence du mouvement de celle-ci sont les effets d'une auto-organisation spontanée, induite dans le cas des poissons, par une communication qui n'est pas chimique mais visuelle. Clairement ces poissons, bien que doués individuellement d'une intelligence très limitée, font preuve dans leur comportement collectif d'une forme évoluée de stratégie de défense. L'intelligence collective dépasse l'accumulation des intelligences individuelles. C'est également le cas, très spectaculaire, des fourmis et notamment des termites, dont les constructions raffinées semblent être basées sur un plan préconçu assez compliqué, puisque dans toutes les termitières d'une même espèce se retrouvent les mêmes structures. Mais aucun des termites ne possède un tel plan. L'architecture émergente est le résultat d'innombrables interactions animées par des règles locales, entre individus, et entre individus et environnement.

Un autre exemple qui a intrigué les biologistes pendant longtemps est l'organisation d'un vol d'oiseaux. Comment expliquer la cohérence et la régularité d'une telle manœuvre collective? L'hypothèse peut-être la plus vraisemblable, celle qui affirme l'existence d'un oiseau meneur, que le reste du groupe suit, a été abandonnée après des études et des observations détaillées. D'autres hypothèses, dont certaines plus compliquées, ont été rejetées. Aujourd'hui, il est généralement admis que les oiseaux ne suivent qu'un nombre très limité de règles élémentaires qui concernent leur voisinage immédiat. On distingue communément une règle de séparation, une règle d'alignement et une dernière de cohésion. Ces trois règles suffisent pour expliquer et simuler la structure émergente. Aucun appel n'est fait à une perception ou connaissance globale que chaque oiseau aurait de la situation spatiale du vol collectif.

\section{VIE ET SOCIÉTÉ ARTIFICIELLES}

Ces exemples montrent la nécessité d'une nouvelle forme de modélisation. Mais par quels moyens les mathématiques peuvent-elles traiter ces comportements 
collectifs, alors que la plupart des objets mathématiques sont de type individuel: un point, une droite, un nombre, etc.? Bien sûr, la théorie des ensembles permet de considérer des groupements d'objets, mais avec le résultat qu'un ensemble d'objets peut être traité à son tour comme objet individuel et ainsi de suite. Nous avons déjà parlé de la statistique, qui permet de transférer des concepts individuels à une collectivité: à partir de la taille des individus d'un groupe, on peut définir une taille «représentative» du groupe, par la notion de moyenne ou par une autre mesure de tendance centrale. Mais pour simuler un ensemble d'individualités, capables de suivre des règles simples pour adapter leur comportement aux particularités de l'environnement et aux rencontres, on peut utiliser la notion d'automate $^{5}$, afin de pouvoir décrire les structures dynamiques émergentes. Cette notion remonte à J. von Neumann, qui a inventé ce concept dans le dessein d'étudier des systèmes composés d'unités simples, mais capables de faire preuve d'un comportement complexe et d'imiter des actions intelligentes. Ces automates, même rudimentaires, peuvent faire preuve d'une conduite collective étonnante, s'ils sont mis en réseau. Ils reproduisent en cela la surprise que provoquent chez nous les insectes sociaux avec leurs constructions subtiles. Ces réseaux d'automates élémentaires, les automates cellulaires et leur généralisation aux systèmes multiagents, fournissent un cadre formel univoque, et rigoureux si on les replace dans le contexte de la physique statistique pour étudier toutes sortes de comportements collectifs. En particulier, ils sont le berceau de la vie et des sociétés artificielles.

C'est probablement par le jeu de la vie, un automate cellulaire de dimension deux, que le mathématicien J. H. Conway a réellement initié les recherches autour de la vie artificielle. Il s'agit d'un réseau rectangulaire de cellules, dont chacune peut être dans l'un de deux états: vivant ou inerte. Le temps est discontinu. Les règles qui régissent ce monde sont particulièrement simples: une cellule vivante reste dans le même état au moment suivant si et seulement si elle a deux ou trois cellules voisines vivantes. Une cellule inerte devient vivante si et seulement si elle a exactement trois cellules voisines vivantes. Ces règles simulent d'une manière stylisée les conditions d'apparition de la vie et de la survie des unités vivantes. Or ce qui pourrait n'être qu'un amusement se révèle comme un univers d'une extraordinaire complexité. Les formes qui émergent semblent posséder leurs propres lois de comportement. Leur étude exige de l'observateur qu'il oublie les lois locales auxquelles obéissent les cellules et invente des concepts nouveaux pour désigner les organismes qui se présentent sous ses yeux. A l'aide de ces concepts, il peut alors construire une théorie qui rend mieux compte des aspects sociaux de cet univers: tout se passe comme si des êtres multicellulaires apparaissaient, se déplaçaient, se combattaient ou collaboraient et cherchaient à trouver des formes de survie adaptées à leurs spécificités. On peut ainsi simuler non seulement un nombre potentiellement infini d'organismes et étudier leurs comportements propres, mais aussi les conditions dans lesquelles ils apparaissent et disparaissent. On voit par exemple que la taille et la recherche de la symétrie ainsi que la densité de «peuplement» jouent un rôle important. Mais la véritable complexité de ce modèle se manifeste par sa propriété d'universalité. On a démontré en effet qu'il

Il s'agit ici d'un concept technique, et non pas de l'idée d'automate comme reconstruction mécanique d'un être vivant. 
existait une configuration de départ, capable d'imiter une machine de Turing, c'est-à-dire d'effectuer, potentiellement, toutes les opérations calculables, soit par un homme soit par un ordinateur. On trouve donc dans l'univers engendré par ce jeu mathématique de la vie, des aspects biologiques, sociaux et cognitifs, qui, même s'ils restent formels, présentent une source d'expérimentation et de questionnement d'autant plus intéressante que les règles locales de ce monde sont simples et entièrement connues.

A première vue, le comportement humain semble échapper totalement à ces modèles issus de la physique décrivant le mouvement des particules. Contrairement à ces dernières, l'homme réagit à des influences intérieures comme des projets, des préférences, des fantaisies et autres, aussi bien qu'à des contraintes extérieures dues aux diverses interactions avec des individus voisins et avec l'environnement. Mais les comportements typiques des foules montrent que malgré la grande disparité de ces influences intérieures, certains traits de ces comportements sont attribuables directement à la collectivité. Dans ces cas, les individus peuvent être assimilés à des particules, du moins en ce qui concerne les traits communs émergents et leur description par des modèles formels. Il n'est pas question de nier l'intentionnalité des actes humains, mais de relativiser son importance dans des situations simples, où les individus et les interactions sont nombreux. Les travaux de Henderson (1971) dans les années 1970 montrent qu'on peut identifier des phases «gazeuses» et des phases «liquides» dans une foule qui se déplace. Un exemple typique se rencontre dans un aéroport lorsque des voyageurs se précipitent dans un hall vers une porte d'embarquement - c'est la phase gazeuse -, où ils se trouvent soudain limités par la place et par la contrainte de se mettre en file - c'est la phase liquide. La théorie cinétique des gaz a permis de construire des modèles mathématiques de ces comportements, et de prévoir leurs points critiques et leurs transitions de phase.

Ces modèles, pour intéressants qu'ils puissent être, ne nous apprennent pas grand chose de nouveau sur le monde social, même si on se limite au mouvement d'une foule. Helbing (1994) décide alors d'intégrer une partie de l'intentionnalité des individus dans son propre modèle du déplacement de piétons. Chaque individu a une direction et une vitesse de déplacement préférées. Il se trouve dans un champ de force. Mais cette préférence se heurte aux comportements des autres piétons et l'oblige à des compromis dans ses déplacements jusqu'au point où la fluidité du trafic global semble satisfaire le plus grand nombre. Les modèles comportent aussi bien des forces attractives, qui maintiennent la cohésion d'un groupe, que des forces répulsives qui poussent les individus à garder leurs distances. Les travaux de Helbing et Molnar (1995) ont montré de passionnants scénarios de mouvement de piétons, lorsqu'on met ceux-ci, par exemple, en présence d'obstacles ou de portes. La panique dans les foules a été l'un des thèmes majeurs de leurs interrogations. Leurs modèles ont été appliqués à des problèmes d'occupation de l'espace et ont permis de décrire des structures spatiales nouvelles dans la création de sentiers qui relient plusieurs bâtiments, par des piétons qui désirent prendre le chemin minimal, mais préfèrent suivre un chemin déjà tracé si celui-ci le mène à peu près dans la bonne direction. Ces idées ont été généralisées par Batty (2003) et appliquées au problème de la croissance urbaine et à d'autres considérations de dynamique géographique, en y incluant 
des notions de la géométrie fractale pour mieux décrire les propriétés des structures émergentes.

La théorie économique est un autre terrain d'essai privilégié de la modélisation mathématique. En particulier en ce qui concerne les concepts collectifs, puisque les phénomènes économiques résultent d'un grand nombre d'interactions non linéaires et se soumettent difficilement à des théories strictement causales. En particulier c'est l'observation et l'étude des cycles qui a été très souvent le point de départ des théories économiques, comme c'était le cas dans l'étude du système solaire. Or les cycles économiques s'avèrent de par leur nature beaucoup plus complexes que les cycles des objets célestes. Les individus qui sont à l'origine des phénomènes économiques observés ne sont pas de simples particules, mais possèdent des intentions et des préférences. Néanmoins, il semble qu'à un niveau supérieur émergent des structures qui possèdent certaines formes d'une régularité, indépendante des contraintes individuelles, dans une très large mesure. De nombreuses interprétations et explications économiques ont été proposées pour rendre compte de la régularité des cycles observés. Et, avec les travaux de Pareto et Walras, est apparue la notion d'équilibre, lorsqu'on attribua aux forces du marché un effet stabilisateur. Mais les processus économiques présentent des phases de ce que l'on appelle aujourd'hui chaos qui se situent en dehors de l'équilibre. Cela implique que, même si les processus étaient déterministes, ils restent largement imprédictibles. Bachelier, un élève de Poincaré, avait proposé, au début du vingtième siècle déjà, de considérer la nature aléatoire des données économiques en introduisant les concepts de bruit et de fluctuation. Le modèle de Bachelier a été perfectionné par Lévy, puis Mandelbrot, qui ont montré que les fluctuations ne sont pas distribuées selon une loi gaussienne, comme le supposait Bachelier.

Pour intégrer dans les modèles économiques les idées de croyance et de choix individuels, Kirman (1994), mais aussi Föllmer ${ }^{6}$, introduisent des agents économiques hétérogènes et aussi la possibilité d'interactions directes entre les agents. Ces modèles, utilisant les concepts mathématiques collectifs issus de la physique, s'éloignent encore d'une description causale des phénomènes. On se tourne en partie vers une économie expérimentale, basée sur l'approche par simulation pour rendre visible les régularités robustes que l'on observe.

Le modèle «Sugarscape» de Epstein et Axtell (1996) fournit un exemple de simulation d'une société artificielle qui intègre l'aspect économique mais aussi de nombreux points de vue biologiques, sociaux et culturels. Ici les différentes interactions permettent de comprendre des mécanismes de structures globales, sans que ceux-ci soient prévisibles a priori. Il faut pour cela recréer des conditions initiales variées et étudier la robustesse et la persistance des phénomènes émergents par des moyens statistiques. Et devant la variété et la complication des structures qui peuvent se présenter et de leur lien non linéaire avec les paramètres, on peut penser à la remarque de $\mathrm{H}$. Simon qui se demandait si les sciences sociales n'étaient pas les véritables sciences «dures » (cité dans Epstein et Axtell 1996). La

Dans «Random economies with many interacting agents », Journal of Mathematical Economics, $\mathrm{n}^{\circ} 1,1974$, p. 51-62. 
complexité inhérente à cet univers n'est pas éliminée, elle ne peut pas l'être, mais elle est apprivoisée (voir Axelrod et Cohen 2000) par l'expérimentation et l'observation, rendues possibles par la simulation. La compréhension des phénomènes collectifs naît d'une recréation très simplifiée du monde, même si ce dernier reste virtuel et modeste. De nombreux processus réels apparaissent dans ce modèle comme l'asymétrie dans la distribution des biens, les migrations, la ségrégation, la coopération, la formation de clans et bien d'autres. Il s'agit d'un véritable laboratoire social, permettant une expérimentation très libre et répétée à volonté.

Ce type d'approche fait suite à des travaux, notamment ceux de Schelling (1978), plus anciens, et d'Axelrod (1984), pour ne citer que ces deux exemples parmi de nombreux autres. Avec des moyens formels rudimentaires - l'informatique n'était pas encore suffisamment développée -, Schelling a étudié l'influence de l'intolérance individuelle sur des phénomènes collectifs de ségrégation. Son constat essentiel est l'impossibilité d'une extrapolation directe. Le comportement collectif n'est pas la somme des comportements individuels. Axelrod s'est penché en particulier sur la diffusion de la culture et les échanges que cela implique. Il s'est intéressé au comportement asymptotique, notamment en ce qui concerne le nombre de régions culturelles différentes qui subsistent à long terme. Au départ, Axelrod considère un modèle de cinq traits culturels, avec chacun dix modalités possibles. Il fait évoluer ce monde virtuel à l'aide de quelques règles locales très simples, comportant un paramètre aléatoire. Si on constate souvent une tendance vers une monoculture, il est intéressant de constater que l'apparition dans certaines conditions d' «îlots culturels » peut empêcher la culture dominante d'envahir toute la société. Le modèle permet d'étudier dans quelles conditions une polyculture peut s'installer et durer. Un résultat important concerne la question de la dépendance de la domination possible d'une culture sur les autres, en fonction du nombre de modalités que peut prendre chaque trait culturel. Plus ce nombre est grand, plus une polyculture a de la chance de s'installer. Et l'on s'aperçoit que le passage de l'un à l'autre ne se fait pas de manière continue. Il s'agit bien d'un phénomène de transition de phase avec une valeur critique séparant les deux domaines. Autour de cette valeur critique, les changements de taille et de nombre des régions culturelles peuvent être rapides et dramatiques. Le modèle, basé sur des concepts collectifs issus de la physique, permet d'étudier les dépendances entre la répartition culturelle initiale et la dynamique de son évolution vers une plus grande diversité ou au contraire vers une monoculture en fonction du nombre de traits et de modalités.

\section{UNE APPROCHE MATHÉMATIQUE «COLLECTIVE»: LA COMPLEXITÉ}

Tous les exemples évoqués dans ce texte montrent des possibilités nouvelles d'appliquer des concepts mathématiques, inspirés de la physique et de la biologie, à ce monde apparemment rétif au traitement formel, des sciences sociales. Les notions collectives, dont nous avons essayé de retracer ici la genèse dans le cadre de la modélisation, ouvrent des perspectives entièrement nouvelles. La recherche 
d'une causalité linéaire, directe, a été abandonnée au profit d'une vision à la fois plus exploratoire et plus globale. Le cadre général de la nouvelle démarche est fourni par la théorie de la complexité, qui n'est pas un champ de connaissances bien délimité, mais un ensemble de disciplines étudiant les phénomènes de l'émergence (voir Mainzer 2004 et Pullman 1996). La modélisation s'attache dans cette perspective à rendre visibles des structures émergentes possédant une certaine persistance, et dont les liens avec les caractéristiques individuelles existent, mais sont complexes et le plus souvent imprévisibles. Ce point de vue ouvre des champs de collaboration entre sciences sociales et mathématiques plus ouverts et plus prometteurs par leur interactivité.

Comme nous l'avons vu, l'application des mathématiques en sciences sociales a parcouru un développement intéressant. Depuis les tentatives d'utiliser un point de vue Newtonien qui retrace les dynamiques individuelles à l'aide d'équations différentielles, jusqu'à la simulation des comportements collectifs dans le cadre des automates cellulaires, on a passé d'une vision mécaniste, déterministe à une approche à la fois plus modeste et plus globale. Et cette modestie cache une ambition nouvelle, celle de recréer un monde artificiel, avec des sociétés virtuelles qui montrent des comportements analogues à ceux que nous observons dans le monde réel. En ce sens, la nouvelle méthodologie peut être une source d'inspiration pour de nouvelles questions et de nouvelles réponses, en même temps qu'elle introduit un aspect expérimental dans un domaine qui semblait voué essentiellement à l'observation. Le rapprochement des sciences sociales et des mathématiques dans cette perspective sera sans doute profitable aux deux disciplines en les forçant à une confrontation de leurs concepts. Sans être une solution à tous les problèmes qui se présentent à nous dans la tentative d'appliquer les méthodes formelles des mathématiques aux sciences de l'homme et de la société, l'approche évoquée ici sera stimulante et peut-être dérangeante. Citons dans cet esprit, et pour terminer, la petite phrase de Edgar Morin: «... la complexité pour moi c’est le défi, ce n'est pas la réponse.» (2005).

Institut de Mathématiques Appliquées

Université de Lausanne

Henri.Volken@unil.ch

\section{RÉFÉRENCES BIBLIOGRAPHIQUES}

Axelrod R., The Evolution of Cooperation, New York, Basic Books, 1984.

Axelrod R. \& Cohen M. D., Harnessing Complexity, New York, Basic Books, 2000.

Batty M., The emergence of cities : Complexity and urban dynamics, preprint 2003.

Condorcet J.-A.-N. de, Esquisse d' un tableau historique des progrès de l' esprit humain, Paris, Flammarion, 1988

Epstein J. M. \& Axtell R., Growing Artificial Societies, Washington, Brookings Institution Press, 1996.

Helbing D., «A mathematical model for the behavior of individuals in a social field», Journal of Mathematical Sociology, n' 19, 1994, p. 189-219.

Helbing D. \& Molnar P., «Social force model for pedestrian dynamics », Physical Review, E 51, 1995, p. 4282-4286. 
Henderson L. F., «The statistics of crowd fluids», Nature, n²29, 1971, p. 381-383.

Kirman A. P., Economics with interacting agents, Santa Fe Institute, 1994.

Mainzer K., Thinking in Complexity. The Computational Dynamics of Matter, Mind and Mankind, New York, Springer, 2004.

Morin E., Introduction à la pensée complexe (nouvelle éd.), Paris, Seuil, 2005, p. 134.

Pullman B., éd., The Emergence of Complexity, Pontifical Academy of Sciences, 1996.

Schelling T. C., Micromotives and Macrobehavior, New York, Norton, 1978.

Thompson D’A. W., Forme et croissance, Paris, Seuil, 1994 (trad. française de On Growth and Form). 